\title{
Icon design and application in $\mathrm{HCl}$
}

\author{
Fu Jiuqiang \\ Beijing Institute of Technology, China \\ fujq0306@126.com
}

Keywords: interface; icon design; icon meaning; elements construction

\begin{abstract}
Icon design is an important part of interface design and it is also valuable manifestation of visualizing phase for interface. Based on the icon design and users perception, this article analyzes the general process of users recognizing icon, propose common icon design factors and find rules of users cognitive effects, which can definitely guide the design practice of future.
\end{abstract}

\section{Introduction}

The definitions of icon can be divided into two levels of broad and narrow: broad sense-icons are graphical symbols for indication, which have features of highly concentrating, conveying information conveniently and remembering easily [1]. This interpretation contains a wide range, including directory signs in the street, traffic signs, toilet signs, Web graphics, software graphics, and even corporate logo. Narrow sense -icon is a language which could be understood by designers and users in the interface. It covers program identification, data identification, selection or ordering, pattern signal or switch command, status indicators, and so on. The icons what this paper describes should be within the narrow sense, which are computer graphics owning clear implications. Desktop icons are software identification and icons in the interface is functional identification.

\section{Software Icons Design Is a Creative Process}

Designing Stage. To fully understand the software icon design, we need to know the stage which icon design is in throughout the software developing process. Taking a small and process-oriented software product as an example, product development could go through the following stages, see Fig.1: 1. Defining product demands. 2. Software design. 3. Product implementing. 4. Product testing. 5. Product releasing. Of course, this is not a simple linear process, but iterated process [2]. Interface design is in the second stage in the software development process, which made up of three parts: the menu design, screen design, and icon design. From this, we can see that the icon design is a necessary part of interface design.

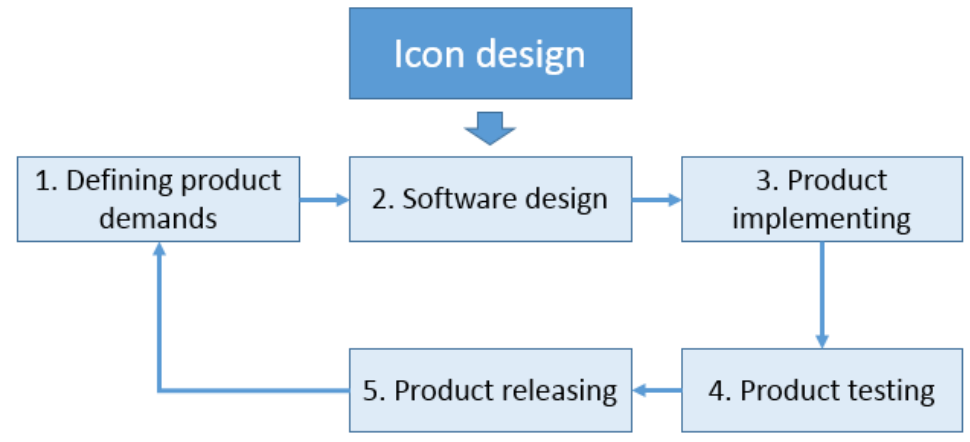

Figure 1. Product development and icon design

A Creative Activity. By analyzing the contents of software interface design and the detailed works of graphic designers, we can understand that software icons design is a creative activity. First, menu design: the menu should provide users with a variety of options related to command and users can use the menus to perform the actions associated with the task [3]. Menu design has considerable limitations, such as the structure and form limitations of operating system. To match for Windows 8 or Mac OS X, 
menus design should meet the specific requirements of each system and there are very strict rules in menu structures, colors and fonts. In this process, therefore, designers have no space to display their creativity. Second, screen design: it involves two problems which can be how to break the task down and put them into multiple screens and how to display certain features in a single screen. What the screen design needed is to have a rational way rather than active creative thinking [4]. Third, icon design: icons are defined as language which designers and users can understand, but whether can it really be understood depends on designers' diving amount [5]. As in the Microsoft Windows operating system, the storage function is expressed by using a floppy disk, and this creates doubt — floppy disks have been largely eliminated in today's computer hardware, why don't you use u disk or hard drive instead? In icon design, designers often encounter similar problems. So they had a lot of interviews, which proved this idea, but floppy disk image was most widely recognized for the international way of expression, and the change like that would violate users ' cognitive habits. Thus interface designers at work often subvert some rules and at the same time should abide a few rules also. During designing, designers will continue to encounter unexpected problems, such as how to express "consumer products in storage list" icon? Facing this situation, interface designers should repeatedly think and constantly brainstorm to find better solutions.

\section{Icon Design Analysis}

As interviewing on some interface designers, when we asked: what is the first consideration about designing icons? Coincidentally, they answered: "how to accurately express the icon's meaning." For example, what kind of widget can be selected to show icons mean? Does split method be used in combination with each other? Are there any industry standards and other issues? Only when these problems are resolved can designers use a pencil sketch project. In this process, the following aspects should be took into account:

Icons and Texts. After designers received the icon design tasks, we recommend that they had better consider whether the software are really in need of icons? Is using words to express better? We often find unnecessary icons appearing in the software, and sometimes the text itself is more persuasive. Solutions to this problem are as follows:

When the interface colors are too rich with complex software content, we do not recommend adding icon.

When the icon meaning is hard to statement by using simple graphic, icons can be replaced by text.

Important function icons should be labeled text below them.

Function icons which require a large sorting or keeping up to date have better be expressed with text as much as possible.

Software Signage and Function Signage. Before designing icon, designers should understand whether icons belong to the software signage or the function signage, and their contents and functions are completely different. Software signage appears on the computer desktop by shortcuts or software logo image, which is similar to the logo of the business or software. Apart from having to consider the overall image of the company and the software, designers also should think over the application scope of the icon, because this kind of icon will not only appear on the computer screen, but also appear in print. The function signage in the software is the expression of some specific function in software. In computer screens, they often appear as buttons. When users select or activate the button, computers will launch the corresponding function.

Composition of the Icon Elements. The composition of icons are divided into two types_-single element and multiple elements. For example, in the Word software, "format painter" is an example of icon consisting of a single element. "Information retrieval" belongs to multiple elements icon, which contains two elements__ a dictionary and a magnifying glass. It is easy for us to understand the meaning of this icon. The dictionary represents carrier of information, the magnifying glass represents the retrieval action, and they together form the "information retrieval" icon. But not all icons meaning can be shown completely, sometimes they are simply representative. For example, if you want to design a "hand-prepared document" icon, first you should think the composition elements of this icon before designing. Because hand-prepared was a very abstract action, 
how can we design to allow users to understand the meaning of the icon? We can expand the divergent thinking from preparation to the things associated need to do it by hand, and hand is indispensable part of the human body, so we can decide that the icon formed by people and a simplified proof. But the meaning of the people element cannot be fully hand-prepared, users may not be able to fully understand that. The best way to solve this problem is labeling text under the icon, and the text description can help users better understand the icon. In addition, the same element might indicate completely different in different icons, such as magnifying glass icon can be interpreted as a zoom function, or can be interpreted as a search function, which requires designers to choose from.

Function Meanings and Text Meanings. What designs should most concern about is that understanding the functional meaning of icons, and correctly interpreting [6]. Understanding the meaning function means that users will realize what kinds of function on the screen after clicking on this button. The functions of icons can be explained in two ways: first is the icon meanings embodied in the text, such as "information retrieval" is a literal meaning; the second is the function software products should owned, which can be interpreted as function features. Function meaning is consistent with the text meaning of the icons sometimes, but sometimes they have entirely different meanings. Designers should pay special attention to their function meaning to express when they have a gap, so that users are not likely to cause unnecessary misunderstanding during operation.

Icon Form Expressions. In the 1990 of the 20th century, the software icon is still belong to the graphic category, as in software running on Windows98, Windows2000 [7]. However, after several years of development, icons expressions widely used a combination of plane and solid method. Icons' perspectives are as follows: plane view icons; two-point perspective view icons; three-point perspective view icon. Features of plane view icons are that these icons represent the object directly in front of it, though it may be have solid preliminary form, but it still only draw a plane effect of three-dimensional object. These icons are most used in $16 \times 16$, or the $32 \times 32$ specifications, and are characterized by a large number of interface using, which are also less prone to confuse because of their smaller size, see Fig.2. For example, most of the toolbar icons in the Office2013 and small icons for setting screen in mobile phones are plane view icons. Two-point perspective view icons are generally $32 \times 32$ or $64 \times 64$, and their functions are activating a function group, so generally speaking their functions are larger than those plane view icons. Three-point perspective software icons typically appear within the key navigation and icons representing the software main features. Icons are made up of elements, and combinations of these elements is regular. Icon designs and paintings are similar, whose screen must have a subject, and designers should add some auxiliary form to subject matter so that the picture will be prioritized. As the "picture" icon in Word software, the icon's body is behind the landscape photos, and the display graphic in front of it is the secondary form.

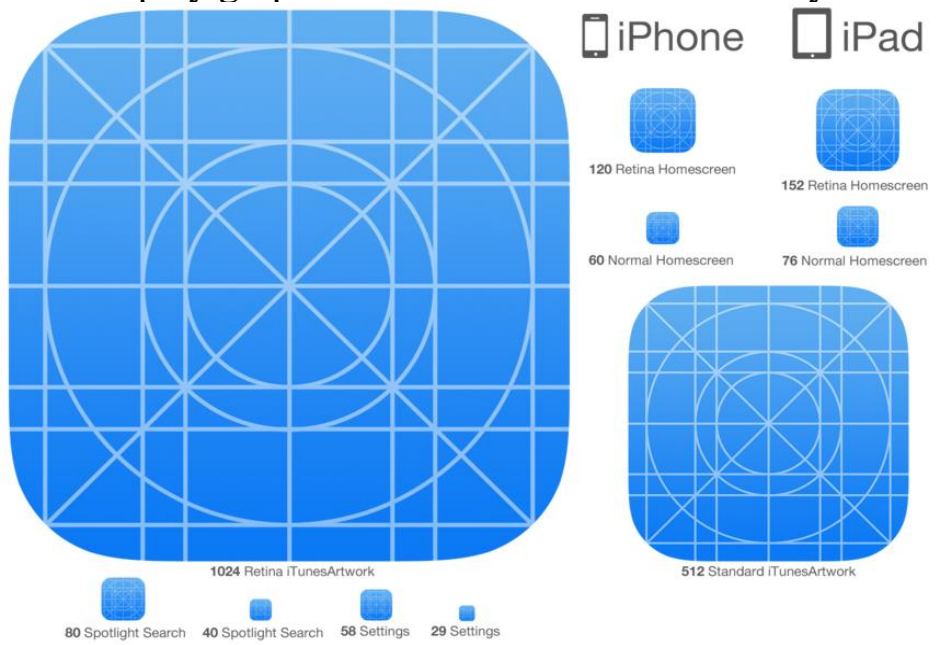

Figure 2. Icon pixel requirement 


\section{Icon Design Guidelines}

Highlighting the Consistency. Icon design's consistency is shown in three aspects: first is that element selection needs consistency. For example, "file" icon uses a sheet of paper to express, "copy" icon uses two sheets of paper to express, and both of them used the paper element. Second, icon's style needs consistency, which can be understood as the icons in the same app with different attributes, such as color, shape, animation, location, etc. Finally, different sizes of the same icon needs consistency. For example, software will have the same icons in several sizes, large icons often are reduced to small icons, which cannot be identified clearly, and at that time they require the designers to adjust, but not spoil consistency.

Allowing Frequent Users to Customize. When the advanced users want to make custom for the interface, they will find that no icon can be used to express some functions. To provide some generic icons in the software could solve this problem. Using generic icons, they can easily find the replacements on the software icon library.

Providing Clear Feedback. When the users operate software, icons as buttons will display the current state of the feature in the interface, and provide clear feedback to users. Icons are always under several conditions: normal display, mouse-over display, click display and the gray display. Designing these types of icon status, designers should focus on drawing clear distinctions.

Error Prevention. In icons designing, to expand appearance differences as much as possible can prevent users identify bugs. The other hand, in terms of location, the icons which have exactly the opposite functions and will cause adverse consequences after clicking should be separated as much as possible.

Reducing the Burden on Memory. If there are more than four elements in an icon, then it will have little recognition. Try to use familiar, or widely used elements to express. If no icon, just remove the icon, the text itself the most convincing.

\section{Conclusions}

Icon design can reflect the general understanding of human being for things, and also can display the concept of social, cultural and others. Modern society is a highly visual society, graphic language, to a large extent, has replaced the traditional language, which can quickly make us finish visual communication. For us, icon design is more than a simple meaning expression of the design process, and it plays an important role in the development process of modern society.

\section{References}

[1] Sarah Isherwood.Graphics and Semantics: The Relationship between What Is Seen and What Is Meant in Icon Design. Engineering Psychology and Cognitive Ergonomics[J]. Lecture Notes in Computer Science Volume 5639, 2009, pp 197-205.

[2] Jacobson, I, Booch, G, Rumbaugh, J.The unified software development process[M]. Addison Wesley, Reading, 1999.

[3] Olson, J. R., Olson, G. M. The growth of cognitive modeling in human-computer interaction since GOMS[J]. Human-Computer Interaction,1990. 5: pp. 221-265.

[4] Debevc, M. 'Adaptive Bar'. INTERCHI '93, Conference on Human Factors in Computing Systems[J], INTERACT '93 and CHI '93, Amsterdam, Netherlands,1993, pp. 117-118.

[5] Kossakowski, M. Adaptive Man-Computer Dialogues. In: Klix, F., Streitz, N.A., Waern, Y., Wandke, H. eds. Man-Computer Interaction Research[J], MACINTER-II. Elsevier Science Publishers, Amsterdam,1989, pp. 201-212.

[6] Preece, J. Human-computer interaction[M]. Addison-Wesley Publishing Company, Workhingham.1994. 